\title{
Agôn
}

Revue des arts de la scène

7| 2015

La Distribution

\section{Yves-Noël Genod, un théâtre de l'invitation}

Entretien réalisé par Isabelle Barbéris

Yves-Noël Genod et Isabelle Barbéris

\section{CpenEdition}

Journals

Édition électronique

URL : http://journals.openedition.org/agon/3350

DOI : 10.4000/agon.3350

ISSN : 1961-8581

Éditeur

Association Agôn

Référence électronique

Yves-Noël Genod et Isabelle Barbéris, "Yves-Noël Genod, un théâtre de l'invitation », Agôn [En ligne], 7 | 2015, mis en ligne le 14 octobre 2015, consulté le 15 septembre 2020. URL : http://

journals.openedition.org/agon/3350

Ce document a été généré automatiquement le 15 septembre 2020.

Association Agôn et les auteurs des articles 


\title{
Yves-Noël Genod, un théâtre de l'invitation
}

\author{
Entretien réalisé par Isabelle Barbéris
}

Yves-Noël Genod et Isabelle Barbéris

\begin{abstract}
Yves-Noël Genod ne se présente lui-même que comme un « distributeur » de spectacle, de poésie et de lumière, il n'invente rien qui n'existe déjà, il fait passer le furet, « passé par ici, il repassera par

là »...
\end{abstract}

Feuille de salle de Rester vivant, Festival d'Automne,

2014.

1 Yves-Noël Genod ne se présente ni comme un "concepteur de performances ", ni comme un "metteur en scène» mais comme un "distributeur de spectacles». L'expression résonne avec certaines acceptions très triviales du terme, allant de la "grande distribution» au «distributeur automatique» (de préservatifs, de friandises...), de l'affichage populiste de la «distribution générale » à l'interlope expertise du «distributeur agréé »... Le « distributeur de spectacle » amène l'industrie de la distribution et de la grande « répartition des richesses » à un point de frottement avec une pratique artistique délibérément mineure, miroitante et fuyante. Adopter la posture du "distributeur de spectacles", c'est redoubler l'horizon d'attente de la démocratisation culturelle et introduire un effet de larsen troublant avec ses propres éléments de langage: nous sommes à l'époque où l'artiste est aidé sur des critères d'efficacité (voire d'efficience) en matière de diffusion et de rencontre avec le public...

2 Une des particularités du travail d'Yves-Noël Genod consiste dans son intense productivité et dans la rapidité du geste de distribution, aussi bien des rôles que des spectacles. Il est capable d'inventer une forme scénique totale et viable en un temps «record» en se jouant de la temporalité de la création scénique. Une intense productivité et, si le mot existait, distributivité (potentiel de distribution)... mais à petite échelle. C'est là où le geste devient complexe, paradoxal, ambivalent: les spectacles (très nombreux, près d'une cinquantaine en une quinzaine d'années) 
tournent pour la plupart assez peu, et disparaissent parfois de la mémoire même de leur auteur, qui se surnomme d'ailleurs lui-même Le Dispariteur

Derrière la formulation en partie provocatrice du «distributeur » de spectacles, on découvre la revendication d'une pratique nomade, qui procède par occupations itératives et mises en volume de lieux souvent atypiques. Distribuer des spectacles revient en effet d'abord à se distribuer dans des lieux et à les investir : point de départ de l'artiste qui se distribue, dans un geste d'auto-dissémination de sa présence et de son image propres, coulant un temps son univers fait de latences et de jeux de superpositions dans des espaces qui sont saisis comme des occasions et qui transforment les spectacles en occurrences : chez Yves-Noël, il n'y a plus de modèle, mais uniquement des exempli, des variations, des choses redistribuées ici et là, sur un théâtre urbi et orbi : rôles, costumes, espaces, éclats de présence et étincelles de poésie...

Plus empiriquement, disons que la distribution n'a chez lui plus grand-chose à voir avec le traditionnel travail de répartition des rôles. Elle s'apparente à une sorte de «tirage au sort » dont l'objet se trouve déplacé : ce n'est pas tant le rôle, ou l'emploi, que Genod distribue mais, bien avant de penser au personnage, le costume et l'enveloppe. Le processus de création ne commence qu'une fois que le costume est porté, et c'est du costume, attribué à un acteur grâce au kairos de la coulisse et de la répétition, que naîtra une dramaturgie scénique qui, idéalement, voudrait substituer au " personnage en costume » ce que Genod appelle des « costumes sans personne dedans ", réactivant ici un lien avec l'antique persona. L'enveloppe que revêt l'acteur est toujours, chez Genod, revendication d'artificialité et sublimation de l'arbitraire. Les séances d'improvisation reposent en grande partie sur la mutualisation d'un fond de costumes que l'artiste transporte de spectacles en spectacles: perruques criardes, habits de lumière un peu élimés mais encore flamboyants une fois léchés par une douche de lumière savamment apprivoisée, chaussures de luxe vintage, papier bulle recyclé en traîne nuptiale, oreilles de lapin ou gants de boxe disposés sur des corps souvent nus, autant d'apparats qui transforment la nudité elle-même en costume, c'est-à-dire en image et en absence. La distribution est donc geste d'absentéisation de la personne de l'acteur au profit du surgissement d'une image ; geste de disparition mis à contribution d'une exaltation du signifiant, de l'enveloppe, autrement dit de ce qui contient plus que de ce qui est contenu, du théâtre (le lieu) plutôt que du drame (son contenu). La modernité du geste $\mathrm{a}$, là encore, beaucoup à voir avec sa rapidité : les costumes se répartissent vite, les jeux de formes et de forces s'esquissent en quelques grands traits de répétition, permettant la création de mini-œuvres d'art total en quelques jours, parfois quelques heures.

5 Genod retourne la notion de « distribution » dans ce qu'elle laisse entendre de coercitif et d'assigné. Son théâtre ne fonctionne pas sur l'assignation mais, comme je l'ai évoqué, sur la latence et la potentialisation : pour l'interprète et concepteur de spectacle passé chez Régy, chaque instant de la représentation se doit de tout contenir afin de ménager toutes les "solutions ", en embrassant le plus de "volume " possible. Contrairement à Régy qui essentialise le vide et y cherche une vérité, l'absentement du signifié vers lequel tend le théâtre de Genod œuvre à la remise en question de l'autorité de l'arbitre - du distributeur. Une dramaturgie qui potentialise autant le jeu de chaque interprète ne peut se réclamer d'une conception assignatrice de la distribution. Bien au contraire, le théâtre commence lorsque l'assignation au rôle, à l'emploi, qui prévaut la plupart du 
temps dans le champ productif de la vie quotidienne, éclate pour laisser place à ce déploiement global des virtualités, qui caractérise chez lui la présence scénique accomplie de l'interprète. Genod inverse la notion de distribution qui recouvre alors celle, glosée par la philosophie de l'émancipation de Rancière, de "partage ». La distribution est un geste de restitution : il s'agit de rendre à l'interprète, au lieu et au public, le théâtre spolié par le morcellement institutionnel... de lui restituer sa capacité d'expression globale et maximale. Pour employer un terme à la mode, la distribution recoupe ici l'empowerment de l'interprète, à qui se trouve remis un éventail de possibles, de préférence au carcan du rôle. Le passage préalable par l'absentéisation sous l'enveloppe précède et engendre l'empowerment de l'interprète : "disparu », il peut faire "n'importe quoi »; dépouillé de son identité, c'est-à-dire de son masque quotidien, il est en mesure de produire des actes purs.

6 En se présentant comme "distributeur de spectacles ", Yves-Noël Genod recourt également à un registre de " discours méta-institutionnel », certes déjà exploré par des artistes tels qu'Antoine Defoort (Un Faible degré d'originalité), Patrick Bernier et Olivier Martin (Projet pour une jurisprudence), Thibaud Croisy (Le Gymnase nihiliste) ou encore par les Gens d'Uterpan dans l'ensemble de leur recherche: ces artistes développent un travail qui entend rejouer, sur un plan artistique, la règle du jeu bureaucratique et en redistribuer la donne, soit en s'y substituant, soit en l'infiltrant et en la parasitant. Cela dit, chez Genod, l'approche se fait sur un mode qui est moins celui de la revendication que celui, comme à son habitude, de l'aparté et du lazzi : Arlequin n'était-il pas déjà le grand "redistributeur» de l'humaine comédie, et celui qui, à l'insu des autres, redistribuait aux personnages les rôles qu'eux-mêmes ignoraient jouer? L'art de Genod nous ramène aux confins du batelage et du théâtre de foire, dans leur fonction primordiale d'exhibition et de redistribution des forces en présence.

7 Marginal, alternatif, parce qu'exhibant l'arbitraire du signe, c'est-à-dire de la distribution du signifiant, l'artificialisme d'Yves-Noël Genod dynamite toute organisation bureaucratique, toute logique institutionnelle de sélection et d'assignation. Subversif, Genod substitue la costumocratie à la bureaucratie : un art de paraître qui, poussé en ses retranchements métaphysiques, s'apparente à une vanité.

Distribuer des apparences et des oripeaux, c'est ce que montre le théâtre de Genod afin de mener le faux et le simulacre, autrement dit l'hypocrisie, à leur point de disparition. Souvent présent dans ses propres spectacles, Le Dispariteur (se) distribue jusqu'à disparaître, rappelant par là la fonction éminemment duplice du geste de distribution, qui met en application l'autorité du metteur en scène " castant » son équipe. Genod en fait un principe de démultiplication de soi et de mise en crise de sa propre autorité. Il réanime volontairement la suspicion qui ombre le statut de l'artiste de tréteaux et de rue : celui qui, de tout temps, distribue son corps, sa voix, ses gestes, qui se distribue lui-même, contre rétribution.

9 Nous l'avons interrogé sur une des spécificités de ses créations, à savoir la présence très fréquente d'invités, distribués dans ses spectacles au gré des circonstances, des amitiés, des occasions et des passages. Ces présences sont en effet emblématiques de l'hospitalité qui préside au geste de distribution dans le travail de Genod. 
Isabelle barbéris. Pour Le Parc intérieur (Festival d'Avignon, OFF, 2010) qui s'est joué à la Condition des soies, il y avait des « guests » qui changeaient chaque jour. Qui étaient-ils ?

Yves-Noël GENOD. Je cherche des interprètes qui ont envie de travailler avec moi juste par liberté, pour le plaisir, à un moment de leur vie (ou bien ça peut durer des années, comme avec Jonathan Capdevielle, Thomas Scimeca, Marlène Saldana...). Ce sont des gens qui n'ont ni à perdre ni à gagner, qui ont - c'est très mystérieux cette disponibilité-là à un moment de leur vie (qui peuvent très bien ne plus l'avoir à d'autres moments de leur vie). Surtout pas pour la carrière, alors, ça ! Ça, je la laisse aux autres... C'est important, pour certains acteurs, sans doute, d'en passer par la carrière. Mais ces interprètes savent (mystérieusement, je le redis) qu'avec moi, on en sera très, très loin, de la carrière, tout à l'inverse même. Il y a un titre de poème de Wallace Stevens que j'aime beaucoup : «Vacances dans la réalité ». C'est un peu ce que je leur propose. Et ce qu'ils comprennent, en tout cas. C'est pour ça qu'on s'amuse. Ce sont des vacances. Dans les stages, pareil. Dans la réalité. Autant que faire se peut. (Par intuition poétique.)

\section{B. Quelle était leur fonction dans le spectacle?}

Y.-N. G. Ils ouvraient le spectacle en servant du champagne au public qui entrait dans la salle et en assurant une courte première partie d'exactement cinq minutes (tout est millimétré dans le Off). J'entrais moi aussi dans la salle au bout de cinq minutes (sans avoir vu la première partie) et je prenais la suite avec le spectacle proprement dit. J'entrais ainsi dans des atmosphères très différentes d'un jour sur l'autre; certaines fois, le public était très fermé, dans l'expectative, d'autres fois très ouvert, dans la joie. J'ai envie de dire qui a rendu l'atmosphère la plus ouverte: Foofwa d'Immobilité. C'était une période de sa vie très heureuse, amoureuse. Quand je suis entré, il finissait de se rhabiller. Il avait dit au public qu'il voulait danser l'amour parce que c'était ce qu'il ressentait et que pour danser l'amour il fallait se mettre nu. Il avait éparpillé ses vêtements partout dans la salle, en hauteur aussi et, à la fin, il demandait au public de lui rapporter ses vêtements. Je lui propose toujours de travailler avec moi, mais il n'est jamais libre, malheureusement.

I. B. Mon impression est que changer d'invité tous les jours attire ton travail vers quelque chose qui relève de la « chronique » et de l'art de saisir le passage...

\section{Y.-N. G. C'est sûr.}

I. B. Peux-tu revenir sur certaines « invitations » (dans ce spectacle ou d'autres), qui ont été particulièrement fécondes et heureuses?

Y.-N. G. Eh bien pour le spectacle intitulé Je m'occupe de vous personnellement, au Théâtre du Rond-Point en juin 2012 (je crois), il y avait aussi des invités qui changeaient tous les jours et qui, eux, étaient inclus dans le spectacle. Le but était de donner un spectacle différent chaque jour. On renommait ainsi le spectacle tous les jours, les textes lus ou appris par Valérie Dréville changeaient tous les jours (mais toujours d'Hélène Bessette), etc. Les gens qui revenaient voyaient à la fois le même spectacle (il y a une force de l'inertie) et pourtant un spectacle complètement mouvant. C'était ça, l'experiment. Et c'est avec ce spectacle que j'ai rencontré, par exemple, certains des interprètes de 1er Avril (2014), les chanteurs Jeanne Monteilhet et Bertrand Dazin qui m'avaient été conseillés par Olivier Martin-Salvan, le trompettiste Louis Laurain, conseillé par Bastien Mignot... 


\section{B. Et des échecs voire des désastres?}

Y.-N. G. Non. Certains des invités sont bien meilleurs que d'autres évidemment. On est déçu, quand même, par certains, mais on est la plupart du temps émerveillé.

I. B. Toi-même dans tous tes spectacles tu accueilles systématiquement les spectateurs à l'entrée de la salle. Les spectateurs sont-ils aussi des «guests»? Font-ils partie du spectacle, du théâtre et de ces espaces que tu crées?

Y.-N. G. Évidemment. Que ferais-je sans eux?

I. B. Tu es toi-même souvent un « invité » : à investir des lieux insolites, de cartes blanches, à "animer» un festival (comme dans le cas de La Poésie la nuit, d'Eric Vautrin). Cela correspond-il à une réalité ?

Y.-N. G. Je suis souvent, même, l'invité surprise, le bouche-trou, pour combler un vide de programmation. Je m'en fiche et même tant mieux parce que c'est comme ça que j'ai joué au Théâtre National de Chaillot, aux Bouffes du Nord, etc. J'ai aussi remplacé Jérôme Bel à la Ménagerie de Verre... Je n'ai pas de blessure avec ça. Claude Régy m'a raconté que pour le premier spectacle que j'ai vu de lui - et qui reste mon préféré Grand et petit (1982), au TNP de Villeurbanne, Michel Bataillon l'avait appelé et lui avait dit tout de go : «Tu n'aurais pas quelque chose? parce qu'on a demandé à tout le monde, partout en Europe, Matthias Langhoff, etc. et personne n'est libre...» Ensuite, pour ce même spectacle créé à Villeurbanne, il a obtenu le Théâtre de l'Odéon, à Paris, parce que le directeur de l'époque était persuadé que Claude Régy était franc-maçon et qu'en le programmant il s'attirerait les bonnes grâces de François Mitterrand qui venait juste d'arriver au pouvoir (etc.).

I. B. Dans le Littré, on trouve cette étymologie possible du verbe «inviter»: du radical sanskrit «vi », aimer, désirer... Ça te parle?

Y.-N. G. Tout est amour.

I. B. Est-ce que tu procèdes à une sélection des acteurs, avec ce que ce mot (qui se cache derrière «distribution ») peut avoir de dur. Par exemple, la question du physique des acteurs, comment l'approches-tu?

Y.-N. G. J'esquive peut-être la question sur le physique parce que je ne la comprends pas. Je ne vois pas ce qu'il y a de dur dans la question du physique. Qu'il y ait des gens beaux et des gens moches, oui, c'est très injuste. Michel Houellebecq en parle beaucoup. Les gens beaux baisent tout le temps et les gens moches jamais. (C'est d'ailleurs à se demander pourquoi, au final, il n'y a pas plus que des gens beaux, depuis le temps que c'est comme ça). C'était ça, la question du physique ? Je ne pense pas. Bien sûr qu'on dit qu'un acteur peut tout jouer. Gérard Depardieu s'était énervé une fois parce qu'un journaliste lui avait dit : « Mais un jockey, vous ne pouvez quand même pas jouer un jockey... » Il avait répondu : «Si, je peux ! C'est très facile à faire, un jockey, ça a un petit pois dans la tête... " Je cite de mémoire, mais c'était à peu près de cet acabit-là. C'est pas gentil pour les jockeys ! Mais très peu de gens savent jouer (parmi les acteurs). Jouer, chez moi, ce n'est pas le problème. Bien sûr : que ceux qui savent jouer jouent! (Valérie Dréville, Jeanne Balibar, Kate Moran, Audrey Bonnet, Nicolas Maury, Jean-Paul Muel...), mais que les autres ne fassent pas semblant de jouer, s'ils n'y arrivent pas, je dirais, naturellement. Je demande aux interprètes de ne jamais tenter autre chose que ce qu'ils savent déjà faire très bien. Que ceux qui savent jouer jouent! Que ceux qui ne le savent pas ne jouent pas! Tu connais la phrase d'Ernesto dans le récit de Duras : «Je ne retournerai plus à l'école [...] parce qu'à l'école on m'apprend des choses que je ne sais pas. » J'adore travailler avec des gens 
qui savent faire des choses, cela dit, comme des chanteurs, des circassiens, des danseurs virtuoses... J'adore la virtuosité... qui concerne mon père. Au moment de 1er Avril, il y en avait deux à qui je ne faisais jamais de notes, c'était Ana Pi (qui s'en plaignait d'ailleurs) et mon père. L'excellence. J'étais soufflé tous les soirs de les voir jouer comme pour la première fois. Chaque soir, je les voyais pour la première fois... oh, mon père, il va bientôt mourir, c'est dommage, un tel savoir qui va se perdre... (Mon père, je l'ai déjà dit, est totalement amateur.)

I. B. II n'y a pas de personnages dans ton théâtre, donc a priori pas de distribution. Comment tu trouves tes acteurs?

Y.-N. G. A priori pas, mais il y a quand même une idée. Ou une envie. Souvent l'idée de partir bien sûr du lieu, mais aussi d'un interprète. Et de choisir ensuite ceux qui feront « distribution " à partir de lui. Dans beaucoup de mes spectacles il y a ainsi un interprète ou deux qui donnent le la, la règle à laquelle les autres devront se soumettre. Par exemple pour Chic by Accident (2012), l'aisance dorée de Charles Zevaco et de Wagner Schwartz à la fluidité et au déshabillé comme poisson dans l'eau a contaminé toute la distribution : il est devenu obligatoire - si on voulait participer au spectacle - de rejoindre cette aisance, cette animalité ou, en tout cas, de s'en approcher.

Oui, il y a une sélection des interprètes, évidemment. Pour certains spectacles dans des lieux prestigieux, la lourdeur de la sélection est en fait d'éliminer - de décourager - ceux qui veulent participer à tout prix. Ainsi pour les Bouffes du Nord. Je ne sais pas dire non. Alors j'ai dit : D'accord, mais ce sera pas payé. Pas du tout. Pas un euro. Mais même avec ce critère, j'ai eu beaucoup de gens et aussi des gens qui se sont faufilés et qui m'ont fait chier. Il y a beaucoup de cinglés chez les acteurs ou, en tout cas, des qui ne savent pas trop ce qu'ils veulent ou, en tout cas, qui ne savent pas qu'ils veulent s'engager pour de mauvaises raisons (le prestige d'un lieu est une mauvaise raison). Je cherche des interprètes qui ont envie de travailler avec moi parce qu'ils n'ont rien à y perdre ni à y gagner, juste l'envie à tel moment de leur vie, juste pour le plaisir (pas pour la carrière) et, bien sûr, ça, ce n'est pas facile (ou de moins en moins facile) car les interprètes savent (de moins en moins ?) ce qu'ils veulent... Ils sont (ou ont toujours été) paumés (mais est-ce que je ne parle pas de moi, là ?)

I. B. Ton travail avec Hervé Le Roux est un compagnonnage très particulier. Comment l'idée t'est-elle venue de mettre sur scène un présentateur de rayonnages croisé dans un supermarché?

Y.-N. G. Sa poésie et son humour m'ont frappé alors qu'il faisait la retape au moment des soldes dans un BHV à peu près désert près de chez moi, avenue de Flandres (et qui a dû fermer depuis). Je n'ai d'abord entendu que sa voix et cet esprit d'une poésie absolument sans violence, sans ambition et je me suis mis à le courser parmi les rayonnages, je me souviens que ça m'a pris un moment car cette voix venait de partout. S'il parlait des nappes anti-moustiques, c'est qu'il devait être au rayon du linge de maison, ou du camping, mais voilà qu'il parlait soudain de rivières de diamant (« au prix du ruisseau »)... (etc.)

I. B. Tu dis pour certains de tes spectacles (Pour en finir avec Claude Régy, 2004 ; Monsieur Villovitch, 2007) que c'est de l'hyperréalisme. Mettre des «vrais gens » sur scène, c'est hyperréaliste. Mais en quoi, par exemple, cela diffère de ce spectacle (Moi, Corinne Dadat, de 
Mohamed El Khatib, 2013) qui met en scène dans un «ballet » une vraie femme de ménage avec un vrai « balai »?

Y.-N. G. J'adorerais être capable de faire des spectacles comme celui avec cette femme de ménage, amener des vrais « corps de métier » sur scène, ce décalage. Je n'y arrive pas, mais j'en souffre. J'en passe toujours par les interprètes professionnels sauf exception comme mon père, par exemple, ou certains amis assez ouverts pour avoir envie de tenter l'expérience. En fait, pour moi, c'est tellement nécessaire que les gens qui participent à ce genre de choses en aient l'envie profonde que ça me paraîtrait une montagne que d'avoir à "pousser » cette envie... D'autres artistes le font que j'admire beaucoup comme Antonija Livingstone qui avait engagé une fois à Belfort un jeune boucher de dix-huit ans qui montrait (c'est un exercice d'école) comment on désosse un lapin... Anjelica Liddel y arrive aussi tellement bien. J'aimerais cette puissance de la rencontre de la vraie vie. Les interprètes, leur mental, me fatiguent un peu en ce moment, ils s'ennuient vite. Je pense que tout le monde s'ennuie vite (je parle de moi, sans doute) à notre époque, mais j'aimerais élargir la fourchette des choix. La rencontre, c'est si rare (dans la vraie vie)... J'ai essayé parfois de proposer à des gens croisés dans la rue de monter sur scène, souvent sans résultat, ils ne viennent pas ou viennent une fois pour voir... Même des gens qui font la manche dans le métro (en chantant, etc.) ne viennent pas. Ils disent que bien sûr ça les intéresse, mais ils ne viennent pas...

I. B. Est-ce que tu serais d'accord avec cette proposition : chez toi, le travail de répétition consiste à définir la distribution, à la laisser venir, à distribuer et répartir les "polarités » dans la création d'un spectacle. On ne répète pas une pièce, on répète une distribution, et puis une fois que «ça se distribue bien », le spectacle est prêt?

Y.-N. G. Absolument. C'est exactement ça. Il n'y a rien à " répéter », mais il faut que les interprètes jouent ensemble. C'est le plus difficile - et de loin. Une fois qu'ils jouent ensemble, ce sur quoi ils travaillent m'importe peu, ils font ce qu'ils veulent. Ce que j'exige (et voilà pourquoi ma tâche est désespérée), c'est qu'ils jouent ensemble. Je me dis que si je leur donnais des tâches (ou des "partitions »), nous y arriverions sans doute mieux. Mais je suis incorrigible : je leur demande tout, tout de suite : la liberté sinon rien. Et la liberté dont on a besoin, quelqu'un l'a dit, ce n'est peut-être d'être enchaîné que par ce qu'on aime...

I. B. Toi-même tu t'es créé une sorte de «personnage public » qui fait qu'on te « distribue » à certaines places. II y a derrière l'idée de distribution quelque chose de brutal: une place assignée (le jeune premier, l'amoureuse, l'amuseur de galerie, etc.). D'une certaine manière, les metteurs en scène sont «distribués» dans le champ des politiques culturelles. Remplacer la distribution par l'invitation (à l'acteur, au spectateur), cela me semble poser la question de la confiance.

Y.-N. G. Eh bien, oui. 